\title{
Erratum to: Modellprädiktive Regelung für das Bandeinfädeln in der Warmwalzstraße
}

\author{
Anastasiia Galkina ${ }^{1}$, Kurt Schlacher ${ }^{1}$ und Ilhom Gafur ${ }^{2}$
}

${ }^{1}$ Institut für Regelungstechnik und Prozessautomatisierung, Johannes Kepler Universität Linz, Linz, Österreich 2Primetals Technologies $\mathrm{GmbH}$, Erlangen, Deutschland

Online publiziert 12. Dezember 2016

\section{Erratum to:}

\section{BHM 2016}

DOI 10.1007/s00501-016-0539-6

Durch ein Versehen wurden die folgenden Korrekturen in der Originalpublikation nicht ausgeführt. Die Fehler wurden inzwischen korrigiert. Der Verlag entschuldigt sich für diese Fehler und die dadurch entstandenen Unannehmlichkeiten.

Die Formel Nummer 8 lautet korrekt

$$
\begin{aligned}
& \min _{z} z^{\top} O z \\
& -W_{\max } \leq e \leq W_{\max } \\
& -U_{\max } \leq u_{\mathrm{k}} \leq U_{\max }, k=2, \ldots, N-1 \\
& -Y_{\max } \leq Y_{\mathrm{k}} \leq Y_{\max }, k=3, \ldots, N \\
& -e \leq W_{\mathrm{k}} \leq e, k=1, . ., N-2 \\
& Y_{N}=Y_{N-1}
\end{aligned}
$$

In dem Text unter der Überschrift „5. Modellprädiktive Regelung für das Bandeinfädeln" wurde eine Textpassage doppelt aufgeführt.

The German version of this article can be found under doi: 10 1007/s00501-016-0555-6

\section{A. Galkina, M.Sc. $(\bowtie)$}

Institut für Regelungstechnik und Prozessautomatisierung,

Johannes Kepler Universität Linz,

Altenbergerstraße 69,

4040 Linz, Österreich

anastasiia.galkina@jku.at 